\title{
Heilsame Achtsamkeit - Gefahr droht durch die digitalen Medien
}

\author{
Urs Hinnena, Gina auf der Maur ${ }^{b}$ \\ ${ }^{\text {a }}$ Dr. med., MPH, Mitglied FMH; ${ }^{b}$ Psychologin, lic. phil.
}

Achtsamkeit gilt heute als bewährtes Mittel zur Förderung der psychischen Gesundheit, indem sie Stress reduzieren hilft und das Burnout-Risiko vermindert, wie vor einer Weile in dieser Zeitung zu lesen war [1]. Bekannt wurde das Konzept der Achtsamkeit des Mediziners Jon Kabat-Zinn durch die von ihm 1979 entwickelte Methode der Mindfulness-Based Stress Reduction zuerst in den USA und schliesslich auch bei uns. Achtsamkeit verhilft dank der damit verbundenen urteilsfreien Wahrnehmung zu einem freundlicheren Umgang mit sich selbst und seiner Mitwelt. Wenn man den Begriff der Achtsamkeit etwas weiter fasst, wird sie zu einer wertschätzenden Hinwendung zu sich selbst und den anderen, also zu einer Gegenspielerin der Gedankenlosigkeit. Gedankenlosigkeit in Bezug auf die Umstände unseres Daseins führt dazu, dass wir Alles als selbstverständlich empfinden, uns nicht bewusst sind, was eigentlich dahinter steckt, wenn es 'rund' läuft, und wir deshalb auch nicht überlegen, was die Auswirkungen des eigenen Tuns auf die Umwelt und die Gesellschaft sind.

Achtsamkeit als Lebenshaltung wird bedroht durch die massiven Umwälzungen des Lebens- und Kommunikationsstils, welchen nicht zuletzt Apple vor zehn Jahren mit der Lancierung des iPhones ausgelöst hat. Die negativen Auswirkungen ständiger Ablenkung durch die digitalen Medien auf die psychische Gesundheit werden immer deutlicher. So stellte die Wirtschaftspsychologin Sarah Diefenbach von der Ludwig-Maximilians-Universität in München fest, dass sich die Wahrnehmung verschiebt: Es zählt nicht mehr der Moment; anstatt das Erlebnis auszukosten und dabei positive Emotionen zu erfahren, befinden sich viele Menschen gedanklich schon im sozialen Netzwerk und überlegen, wie sie das Erlebte dort präsentieren. Im sozialen Netzwerk reiht es sich dann ein neben den Erlebnissen der anderen. Der Vergleich führt unweigerlich zu Unzufriedenheit, weil das Leben der anderen aufregender und glücklicher wirkt als das eigene [2].

Da wir im Arbeitsalltag immer wieder mit Patienten konfrontiert sind, welche sich selbst nicht mehr wahrnehmen, sollten wir über ein grundlegendes Verständnis darüber verfügen, wie wir diesen Menschen zu mehr Achtsamkeit verhelfen können. Als ersten Schritt sollten wir uns selbst Gedanken darüber ma- chen, 'was wirklich ist'. Hierzu ist explizites Denken gefragt [3]. Werden wir uns also über das 'Wesen' unserer Existenz so weit wie möglich klar, indem wir 'die Brücke zwischen Kultur und Natur begehen'. Das bedeutet, dass wir unser Wissen über die Evolution 'nicht einfach mitlaufen' lassen. Der heutige Mensch stellt das Produkt einer 4 Milliarden Jahre dauernden Entwicklungsarbeit dar. Damit steigt die Wertschätzung uns und der Mitwelt gegenüber deutlich. Sind wir selbst dankbar für das, was unser Körper tagtäglich leistet? Um unser eigenes Tun immer wieder zu reflektieren, können wir uns der exzentrischen Positionalität bedienen - eines Konzeptes, das der deutsche Soziologe Helmut Plessner entwickelte. Die Möglichkeit des Wechsels zwischen zentrischer (auf sich selbst ausgerichteter) und exzentrischer Position (Wahrnehmung der eigenen Position von aussen, gewissermassen aus der Sicht der anderen) ist einzigartig und wohl nur uns Menschen gegeben [4]. Dank dieser Fähigkeit können wir uns jederzeit durch Einnahme einer exzentrischen Position von aussen betrachten, und somit von uns bzw. unseren aktuellen Problemen distanzieren. Distanzierung ist eine wirksame Möglichkeit zur Stressbewältigung [z.B. 5]. Nach einer Phase des Übens schaffen wir uns auf diese Weise besonders in Stresssituationen Gelegenheiten, explizites Denken anzuwenden, die Achtsamkeit hervorzulocken und der Situation aus einer anderen Warte zu begegnen.

Um den mit den neuen Medien einhergehenden Gefahren für die psychische Gesundheit zu begegnen, können wir etwas tun: Machen wir uns und unsere Patienten immer wieder darauf aufmerksam, dass es etwas gibt, was uns gut tut - den Ausschaltknopf am mobilen Gerät drücken und bewusst erleben, welches Spektakel (explizit gedacht) sich in jedem Moment unseres Daseins abspielt!

\section{Literatur}

1 Adrian Ritter. Heilsame Achtsamkeit. Schweiz Ärztezeitung. 2016;97(38):1330-2.

2 Sarah Diefenbach, im Gespräch mit Angela Barandun. TA 30.12.2016, S. 2-3.

3 Peter Sloterdijck. Du musst dein Leben ändern. Suhrkamp Frankfurt am Main, 2009:17-19,25.

4 Andreas Heinz. Der Begriff der psychischen Krankheit. Berlin: Suhrkamp Taschenbuch Wissenschaft. 2. Auflage 2015: 109, 223-7, 311

5 Tobias Knecht. Das transaktionale Stressmodell von Richard Lazarus. GRIN Verlag, 2011. 